\title{
Frequency of Chlamydia Trachomatis in Symptomatic and Hsymptomatic Non-pregnant Women in Golestan Province
}

\section{Samira Okhli (MSc)}

Department of Microbiology, Islamic Azad University, Ashkezar Branch, Yazd, Iran

Shaghayegh Anvari(PhD)

Laboratory Sciences Research Center, Golestan University of Medical

Sciences, Gorgan, Iran

Aylar Jamali(PhD)

Laboratory Sciences Research Center,

Golestan University of Medical

Sciences, Gorgan, Iran

Mohammad JavadKazemi(PhD) Department of Microbiology, Islamic Azad University, Ashkezar Branch,

Yazd, Iran

Corresponding Author: ShaghayeghAnvari

Email: shaghayeghanvari@yahoo.com

Tel: +989124026294

Address: Department of

Microbiology, Golestan University of

Medical Sciences, Gorgan, Iran

Received : 02 Jun 2014

Revised: 30 Agu 2014

Accepted: 08 Sep 2014

\section{ABSTRACT}

Background and 0bjective: Chlamydia trachomatis is one of the most common causes of genital infection in men and women. Genital chlamydial infections in women are clinically asymptomatic in 70-80\% of the cases; therefore, the lack of timely diagnosis and treatment leads to complications such as infertility and ectopic pregnancy. The aim of this study was to evaluate the frequency of chlamydial infection in symptomatic and asymptomatic women in the Golestan province.

Methods: This cross-sectional study was conducted on 150 cervical swab samples obtained from 150 women referred to the clinic, after obtaining written consent and completion of questionnaires. The swab samples were transferred to laboratory in phosphate-buffered saline solution and INA extraction was carried out using phenolchloroform and boiling methods. The frequency of chlamydial infection was evaluated by PCR.

Results: None of the tested samples were found as Chlamydia-positive.

Conclusion: The findings require that some more extensive research with larger sample sizes and dispersed population be performed to determine the true prevalence. Considering the serious complications of chlamydial infections and its asymptomatic nature, a highly sensitive and specific method such as PCR should be used to detect Chlamydia. It is suggested that this method be used along with a complementary test to obtain the results that are more accurate. Furthermore, conducting simultaneous studies on other populations at risk will be very helpful in obtaining representable national data.

Keywords: Chlamydia Trachomatis, PCR, Vaginal Swab.

This paper should be cited as: Okhli S, Anvari SH, Jamali I, JavadKazemi M. [Frequency of Chlamydia Trachomatis in Symptomatic and Asymptomatic Non-pregnant Women in Golestan Province]. mljgoums. 2016; 10(2):8 -13 


\section{INTRODUCTION}

Chlamydia trachomatis is a small coccoid, Gram-negative and immobile bacterium living as an obligate intracellular parasite of humans and animals (1). C. trachomatis is one of the most common causes of sexually transmitted diseases (STD) in men and women that are treatable (2).

According to the World Health Organization, approximately 92 million new cases of chlamydial infection occurs in the world, and 4-5 million of these new cases happen only in the United States (3).

Chlamydia causes nongonococcal urethritis and epididymitis in men, Reiter's syndrome or proctitis and conjunctivitis in men and women, and cervicitis, urethritis, endometritis, salpingitis and perihepatitis in women (4).

More than half of $C$. trachomatis infections are asymptomatic in both men and women. If not treated properly, they can lead to severe complications such as pelvic inflammatory disease, ectopic pregnancy and infertility in women (2).

The C. trachomatis infection in cervix may be transmitted to the baby during childbirth, which leads to pneumonia and conjunctivitis in newborns (4).

The prevalence of $C$. trachomatis infection has been estimated $6.5 \%$ to $25 \%$ in Iran and other countries (5). Moreover, the prevalence of asymptomatic infections in other countries has been reported between $1.6 \%$ and 19\% depending on the population studied and the methods used. These individuals are permanent reservoirs for infection due to lack of treatment; therefore, it is necessary to stop the transmission chain for prevention and infection control. An important step to achieve this goal is to identify infections in asymptomatic and symptomatic patients (6).

In addition to adverse social effects, the cost of treatment is high. The annual cost of treating the complications caused by $C$. trachomatis infections in the United States is estimated to be over $\$ 2$ million (5).

C. trachomatis specie has 19 serotypes (K-A, L1, L2, L2a, L3, Ba, Ga, Da, and Ia) that are identified by polyclonal and monoclonal antibodies against the outer membrane protein (MOMP) (7).

MOMP is coded by the ompl gene and is the immunodominant antigen of $C$. trachomatis that acts as a cytoadhesion by facilitating the interaction between bacteria and host cells. It has been proven that MOMP is also involved in adhesion mechanisms and pathogenesis (8). For many years, isolation of bacteria in tissue culture usually adjacent to McCoy cells or cyclohexamide was the method of choice for detection of C. trachomatis (9).

However, this method requires accurate sample collection and transfer conditions and 48 to 72 hours to run (2). The molecular genetic techniques are useful for identification of microorganisms that are difficult to cultivate or grow slowly (such as $C$. trachomatis) (10).

Recently, the polymerase chain reaction (PCR) method is used for the diagnosis of $C$. trachomatis infections. The previous studies show that PCR method has $97 \%$ to $100 \%$ sensitivity and $98 \%$ specificity for the detection of $C$. trachomatis, while cultivation has $85 \%$ and $100 \%$ sensitivity and specificity, respectively (2). The DNA amplification techniques are used for urine samples in addition to cervical and vaginal swabs (11).

A compiled program to recognize the status of the pathogen in different social groups, to identify risk factors predisposing to the infection and to perform screening programs can reduce the burden of this disease in our society.

Considering the complications of chlamydial infection in women, this study aimed to determine the prevalence of infection in symptomatic and asymptomatic women using a sensitive molecular method.

\section{MATERIAL AND METHODS}

This study was conducted on 150 nonpregnant women referring to gynecological clinic of SayyadShirazi Hospital in Gorgan.

The participants were categorized in the group with genital symptoms based on physical examination by a physician and questionnaire information, if they had at least one or more of the following clinical symptoms: abnormal vaginal discharge, spotting, bleeding after intercourse, pain or burning during urination, lower abdominal pain and painful intercourse (dyspareunia). The women with none of the mentioned symptoms, admitted for other reasons, were placed in the asymptomatic group.

The inclusion criteria were marriage, reproductive age and nulliparity. The eligible placed in the asymptomatic group. 
The inclusion criteria were marriage, reproductive age and nulliparity. The eligible individuals were recruited in the study after receiving brief explanation about the study. After obtaining written consent from all participants, they were interviewed and completed a previously designed questionnaire consisting of information about age, ethnicity, and type of clinical symptoms indicative of infection.

The samples were obtained by cervical swab and kept in $2 \mathrm{ml}$ of phosphate-buffered

They were then quickly transferred to the Microbiology laboratory of Golestan University of Medical Sciences on the same day.

To extract the DNA ,two methods of phenolchloroform and boiling were used.

One $\mathrm{ml}$ of the sample solution was centrifuged at $8000 \mathrm{rpm}$ for 10 minutes. The supernatant was removed and $600 \mu \mathrm{l}$ of lysis buffer (EDTA-NaCl, Tris), $13 \mu \mathrm{l} 10 \%$ SDS and $5 \mu \mathrm{l}$ proteinase $\mathrm{K}$ were added to the sediment. The sample was kept at $60{ }^{\circ} \mathrm{C}$ for one hour.

Phenol-chloroform at the same volume of sample solution was added to DNA lysate and then slowly mixed. The mixture was centrifuged at $10000 \mathrm{rpm}$ for 10 minutes and then supernatant was transferred to a new microtube.

Next, $500 \mu \mathrm{l}$ of $7.5 \mathrm{M}$ sodium acetate and $2 \mathrm{ml}$ of absolute ethanol were added and the tube was place at $-70{ }^{\circ} \mathrm{C}$ for 10 minutes. Then, it was centrifuged again for 30 minutes at 10000 rpm.

The supernatant was removed and the sediment was washed with $70 \%$ ethanol and the microtube was placed to dry up. Finally, $10-50 \mu 1$ of the distilled water were added to the mirotube and then it was kept at $-20{ }^{\circ} \mathrm{C}$ until the PCR experiment.

First, the solution containing samples was vortexed and $400 \mu \mathrm{l}$ transferred to a microtube. The microtube was centrifuged at $13000 \mathrm{rpm}$ for 30 minutes. The supernatant was removed and $100 \mu \mathrm{lTris}-\mathrm{Hcl}(10 \mathrm{mM})$ with $\mathrm{pH} 7.5$ added to the sediment. The microtube was stored at $-70{ }^{\circ} \mathrm{C}$ for 48 hours.

After melting, it was boiled for 10 minutes at $100{ }^{\circ} \mathrm{C}$ and then the mixture was centrifuged for 2-6 minutes at $10000 \mathrm{rpm}$. The supernatant containing DNA was transferred to another microtube and kept at $-20{ }^{\circ} \mathrm{C}$ until the PCR experiment. Quality and quantity of the extracted DNA through both methods were assessed using spectrophotometry and electrophoresis on agarose gel (1\%).

PCR process The following omp-1-specific primers were used for the PCR $(12,13)$ :

ompl-F: 5'-GCC-GCT-TTG-AGT-TCT-GCTTCC-TC-3'

ompl-R: $\quad 5^{\prime}$-ATT-TAC-GTG-AGC-AGC-

TCT-CTC-AT-3'

PCR was performed using Bioflux kits at volume of $50 \mu \mathrm{l}$. The PCR buffer (10X), $\mathrm{MgCl}_{2}(50 \mathrm{mM})$, dNTP $(10 \mathrm{mM})$, forward and reverse primers at concentration of $30 \mathrm{pm}$, Taq DNA polymerase $(1 \mathrm{U} / \mu \mathrm{l})$ and deionized distilled water were used. The PCR programs included five-minutes at $95{ }^{\circ} \mathrm{C}, 1$ minute at 95 ${ }^{\circ} \mathrm{C}, 1$ minute at $55{ }^{\circ} \mathrm{C}$ and 90 seconds at $72{ }^{\circ} \mathrm{C}$ over 40 cycles.

The PCR products were electrophoresed on $1.5 \%$ agarose gel stained with ethidium bromide, and then examined under UV light. Along with each PCR run, a standard positive control sample (F/IC-CAL3) and negative control were included to verify the accuracy of the PCR run.

\section{RESULTS}

The age range of the participants was 18 to 60 (mean age 37). The demographic and clinical characteristics are presented in Table 1.

Table 1- Demographic and clinical characteristics of subjects

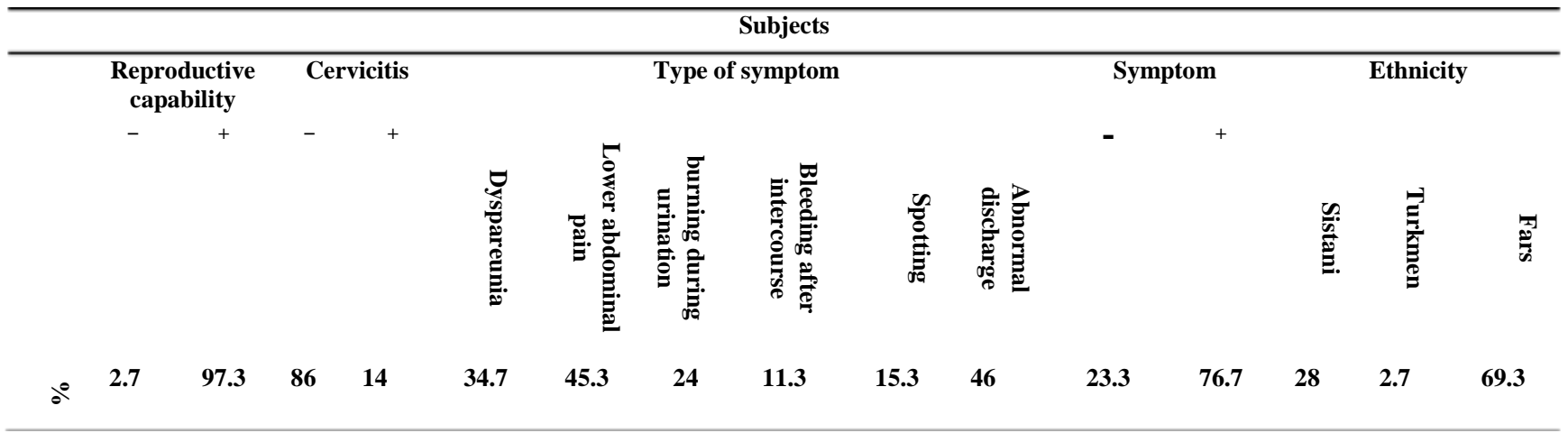




\section{DISCUSSION}

C. trachomatis is one of the most common causes of STDs in the world (3). Since 50\% of infected men and $80 \%$ of infected women are asymptomatic, the actual number of reported cases represents only part of the population infected with Chlamydia, which can make detection and diagnosis difficult (7). On the other hand, lack of timely diagnosis and treatment can lead to ascending infections, causing inflammation and scarring in the reproductive system of men and women. Many reports have shown that these infections are the major cause of pelvic inflammatory disease (PID) in women. One of the most important complications of PID is infertility due to obstruction of fallopian tubes, ectopic pregnancy and chronic pelvic pain (14). In many countries, the incidence of ectopic pregnancy is increasing, which is the main cause of maternal mortality in the first trimester of pregnancy. In addition, Chlamydia can be transmitted to the baby at birth and leads to pneumonia and conjunctivitis (14). The untreated men are also at risk of urethritis, proctitis, epididymitis or epididymo-orchitis (3). In addition to high cost of treatment, the complications of chlamydial infection can sometime lead to some social problems in families. Since the symptoms are not specific to $C$. trachomatis infections, the definitive diagnosis of this infection requires laboratory methods (15). For this purpose, the present study assessed the frequency of $C$. trachomatis in symptomatic and asymptomatic women. Since this was an epidemiological study, the choice of sensitive diagnostic method for determining the prevalence was very important. Several studies have been conducted in recent years in order to find the best method of diagnosing chlamydial infections. Since Chlamydia is an intracellular pathogen, its identification using routine methods of bacteria detection is difficult. Several studies have shown that urine and swab samples can be used for screening $C$. trachomatis as the gold standard for diagnosing chlamydial infections, due to the high sensitivity and specificity of DNA amplification techniques. In addition to low cost of these techniques for testing a large number of samples, it is sensitive enough to a small number of bacteria, PCR with high sensitivity is capable of detecting even small number of bacteria and determining the prevalence of infection in these individuals (17). Several studies in other countries investigated the prevalence of chlamydial infections using different methods by considering their sensitivity and specificity. Goulet study on the general population of France, aged 18-44, reported the prevalence of this infection $1.4 \%$ in men and $1.6 \%$ in women using the PCR method (18). Using the same method, the frequency of $C$. trachomatis in endocervical samples was $9.2 \%$ in India (20). The highest rate of infection $(27 \%)$ was observed in New Guinea (21), while its prevalence was reported $1.1 \%$ in Joyee study in India on urine samples of women using PCR (22). The results of the present study are in agreement with Deeb et al. study. Although in this study the prevalence of other STDs was $1.2 \%$, no positive case of chlamydial infection was found (23). Studies of different groups in Iran reported different frequencies for these infections. In a study in Tehran, the prevalence of $C$. trachomatis in symptomatic and asymptomatic women was reported $14.99 \%$ (24). While, another study on women aged 1542 in Tehran reported that $12.8 \%$ had positive PCR test (16). Moreover, a study reported that $13.2 \%$ of women with spontaneous abortion were infected with $C$. trachomatis (5). The prevalence in Ahwaz was $18.1 \%$ in a study on cervical samples from symptomatic women using the ompl gene (12). In the study conducted by Nasrollahi, the prevalence in asymptomatic and symptomatic women was $16 \%$ and $45.2 \%$, respectively (25).

In Kalantar et al. study in Yazd, no positive cases of $C$. trachomatis infection was found among blood and vaginal samples of infertile women using PCR and ELISA methods (15). The difference between these results could be due to diagnostic methods, different sample type and methods of processing and preparation (26). In this study, the highly sensitive PCR method was applied using ompl gene to obtain reliable results. Two methods of phenol-chloroform and boiling were used for DNA extraction from cervical swab samples but no positive case of $C$. trachomatis infection was found. 
detect Chlamydia (16). Given that asymptomatic infections are accompanied with

\section{CONCLUSION}

Considering the serious complications of chlamydial infections and its asymptomatic nature, a highly sensitive and specific method such as PCR should be used to detect

\section{REFERENCES}

1. Rashidi B,Chamani Tl, Haqollahi F, Ramezanzadeh F,Shariat M,Rahimi Foroushani A, et al. Prevalence OfChlamydia TrachomatisInfectionInFertile And Infertile WomenBySerologicAnd MolecularMethods.Journal Of Reproduction \& Infertility. 2007;4:32-41.[persian]

2. Fallah F, Kazemi B, Goudarzi H, Badami N, Doostdar F, Ehteda A, et al. Detection of Chlamydia trachomatis from urine specimens by PCR in women with cervicitis. Iranian Journal of Public Health. 2005;34(2): 20-26.

3. Meidani M, Chamani Tabriz L, Zeraati H, Razin B, Jamali B, Gachkar L, et al. Molecular assessment on the prevalence of urogenital infection with chlamydia trachomatis in asymptomatic men. Journal of Isfahan Medical School. 2008;25(87):45-53.[persian]

4. Chernesky MA. The laboratory diagnosis of Chlamydia trachomatis infections. The Canadian Journal of Infectious Diseases \& Medical Microbiology. 2005;16(1):39-44

5. Zahirinia Z, Eslami G, Goudarzi $\mathrm{H}$, Taheri S, et al. Prevalence of Chlamydia trachomatisinfectionofspontaneous abortionreferred to ShahidBeheshticenters byNested PCRmethodin 2013. Journal of Research inMedical. 2013;31(1):67-72. [persian]

6. Lan J, Melgers I, Meijer C, Walboomers J, Roosendaal $\mathrm{R}$, Burger $\mathrm{C}$, et al. Prevalence and serovar distribution of asymptomatic cervical Chlamydia trachomatis infections as determined by highly sensitive PCR. Journal of clinical microbiology. 1995;33(12):3194-7.

7. Hsu M-C, Tsai P-Y, Chen K-T, Li L-H, Chiang C-C, Tsai J-J, et al. Genotyping of Chlamydia trachomatis from clinical specimens in Taiwan. Journal of medical microbiology. 2006;55(3):301-8.

8. Nunes A, Borrego MJ, Nunes B, Florindo C, Gomes JP. Evolutionary dynamics of ompA, the gene encoding the Chlamydia trachomatis key antigen. Journal of bacteriology. 2009;191(23):7182-92.

In another study, the frequency of $C$. trachomatis in women aged 15-24 in Brazil was $9.8 \%$ using PCR (19).

9. Schachter J, Stamm WE, Quinn TC, Andrews WW, Burczak JD, Lee HH. Ligase chain reaction to detect Chlamydia trachomatis infection of the cervix. Journal of clinical microbiology. 1994;32(10):2540-3.

10. Bass C, Jungkind D, Silverman N, Bondi J. Clinical evaluation of a new polymerase chain reaction assay for detection of Chlamydia trachomatis in endocervical specimens. Journal of Clinical Microbiology. 1993;31(10):2648-53.
Chlamydia.

\section{ACKNOWLEDGMENTS}

The author would like to thank the staff of SayyadShirazi Hospital in Gorgan for their cooperation.

\section{CONFLICT OF INTEREST}

The authors have no conflict of interests.

11. Jaton K, Bille J, Greub G. A novel real-time PCR to detect Chlamydia trachomatis in first-void urine or genital swabs. Journal of medical microbiology. 2006;55(12):1667-74

12. Taheri BB, Motamedi H, Ardakani M. Genotyping of the prevalent Chlamydia trachomatis strains involved in cervical infections in women in Ahvaz, Iran. Journal of medical microbiology. 2010;59(Pt 9): 1023-8. doi: 10.1099/jmm.0.016717-0.

13. Ngandjio A, Clerc M, Fonkoua MC, Thonnon J, Lunel F, Bébéar $\mathrm{C}$, et al. Restriction endonuclease patterns of the ompl gene of reference Chlamydia trachomatis strains and characterization of isolates from Cameroonian students. Journal of medical microbiology. 2004;53(1):47-50.

14. Watson EJ, Templeton A, Russell I, Paavonen J, Mardh P-A, Stary A, et al. The accuracy and efficacy of screening tests for Chlamydia trachomatis: a systematic review. Journal of medical microbiology. 2002;51(12):1021-31.

15. Kalantar SM, Kazemi MJ, Sheikhha MH, Aflatoonian A, Kafilzadeh F. Detection of Chlamydia trachomatis infection in female partners of infertile couples. Iranian Journal of Fertility and Sterility. 2007;1(2):79-84.

16. Chamani TL, jedi TM, Zeraati H, Asgari S, Moini M. A molecular survey of chlamydia trachomatis infection in married women: a cross sectional study on 991 women.Tehran University Medical Journal (TUMJ). 2008;66(7): 485-491.

17. Sturm-Ramirez K, Brumblay H, Diop K, GuèyeNdiaye A, Sankalé J-L, Thior I, et al. Molecular epidemiology of genital Chlamydia trachomatis infection in high-risk women in Senegal, West Africa. Journal of clinical microbiology. 2000;38(1):138-45.

18. Goulet V, de Barbeyrac B, Raherison S, Prudhomme M, Semaille C, Warszawski J. Prevalence of Chlamydia trachomatis: results from the first national populationbased survey in France. Sexually transmitted infections. 2010;86(4):263-70.

19. Pinto VM, Szwarcwald CL, Baroni C, Stringari LL, Inocêncio LA, Miranda AE. Chlamydia trachomatis prevalence and risk behaviors in parturient women aged 15 to 24 in Brazil. Sexually transmitted diseases. 2011;38(10):957-61. 10.1097/OLQ.0b013e31822037fc.

20. Sood S, Mukherjee A, Bala M, Satpathy G, Mahajan $\mathrm{N}$, Sharma A, et al. A pilot study for diagnosis of genital Chlamydia trachomatis infections by polymerase chain reaction among symptomatic Indian women. Indian Journal of Dermatology, Venereology, and Leprology. 2012;78(4):443. 
21. Passey M, Mgone C, Lupiwa S, Suve N, Tiwara S, Lupiwa T, et al. Community based study of sexually transmitted diseases in rural women in the highlands of Papua New Guinea: prevalence and risk factors. Sexually transmitted infections. 1998;74(2):120-7.

22. Joyee A, Thyagarajan S, Rajendran P, Hari R, Balakrishnan P, Jeyaseelan L, et al. Chlamydia trachomatis genital infection in apparently healthy adult population of Tamil Nadu, India: a population-based study. International journal of STD \& AIDS. 2004;15(1):51-5.

23. Deeb ME, Awwad J, Yeretzian JS, Kaspar HG. Prevalence of reproductive tract infections, genital prolapse, and obesity in a rural community in Lebanon. Bulletin of the World Health Organization. 2003;81(9):639-45.
24. Fatholahzadeh B, Bahador A, Hasanabad MH, Bazarjani F, Haghighi F. Comparative screening of Chlamydia trachomatis infection in women population in tehran, iran. Iranian Red Crescent medical journal. 2012;14(5):289.

25. Nasrolahei M, Sharif M, Robson H. Comparison of pcr based assay and cell culture in detection of urogenita $l$ chlamydia trachomatis infection and determination of the inhibitory rate of specimens under study. Medical Journal of The Islamic Republic of Iran (MJIRI). 2003;17(3):239-44.

26. Shafer MA, Moncada J, Boyer CB, Betsinger K, Flinn SD, Schachter J. Comparing first-void urine specimens, self-collected vaginal swabs, and endocervical specimens to detect Chlamydia trachomatis and Neisseria gonorrhoeae by a nucleic acid amplification test. Journal of clinical microbiology. 2003;41(9):4395-9. 